\title{
Le modèle de turbulence homogène et son utilité pour la compréhension du phénomène turbulent
}

\author{
Homogeneous turbulence and its usefulness \\ in understanding the turbulent phenomenon
}

\author{
J.N. Gence \\ Professeur à l'Université de Lyon 1
}

\begin{abstract}
L'intérêt essentiel du modèle de turbulence homogène est de permettre l'isolation de certains mécanismes typiques d'interaction entre champs moyens et fluctuants et de fournir un cadre de description permettant l'analyse de Fourier. Ce modèle que l'on sait très bien approcher expérimentalement est la base des développements théoriques les plus élaborés concernant les écoulements turbulents. Il a permis, à l'aide d'expériences de laboratoire ou de simulations numériques directes, l'étude et la modélisation des transferts des grosses vers les petites échelles ainsi que de l'action, sur le mouvement fluctuant, de divers champs moyens à gradient constant dont la distorsion pure, le cisaillement et plus récemment la rotation de solide.
\end{abstract}

The main concern relating to this homogeneous turbulence model is to isolate certain typical mechanisms concerning interactions between average and fluctuating fields and to provide a descriptive context enabling Fourier's theory to be analysed. This model, currently being approached in a highly experimental way, is the basis of the most advanced theoretical developments concerning turbulent flows. By means of laboratory experiments or direct numerical simulations, this model has allowed for the study and modeling of transfers of large scales to small scales, as well as the action, as regards the fluctuating movement, of various average constant gradient fields, including pure distorsion, shearing and, more recently, solid rotation.

Raison d'être du modèle de turbulence homogène

L'une des difficultés de l'étude des écoulements turbulents menée dans le contexte de la décomposition de Reynolds

$$
U_{i}(\vec{x}, t)=\bar{U}_{i}(\vec{x}, t)+u_{i}(\vec{x}, t)
$$

réside dans le fait que, dans le cas général, les champs moyens et fluctuants sont en interaction. Par suite, toute tentative d'explication et de modélisation du comportement du champ fluctuant ne peut à priori s'affranchir de l'évolution spatiale du champ moyen et inversement. Par exemple, l'équation d'évolution des composantes $\bar{U}_{\mathrm{i}}$ s'écrit

$$
\begin{aligned}
& \frac{\partial \bar{U}_{i}}{\partial t}+\bar{U}_{i} \frac{\partial \bar{U}_{i}}{\partial x_{i}} \\
& =-\frac{1}{\rho} \frac{\partial \bar{P}}{\partial x_{i}}+v \frac{\partial^{2} \bar{U}_{i}}{\partial x_{k} \partial x_{k}}-\frac{\partial}{\partial x_{i}} \overline{u_{i} u_{i}}
\end{aligned}
$$

et fait donc apparaitre l'influence du champ fluctuant par le gradient du tenseur de Reynolds $\overline{u_{i} u_{i}}$. De même l'équation du tenseur de Reynolds contient des termes liés au champ moyen. 


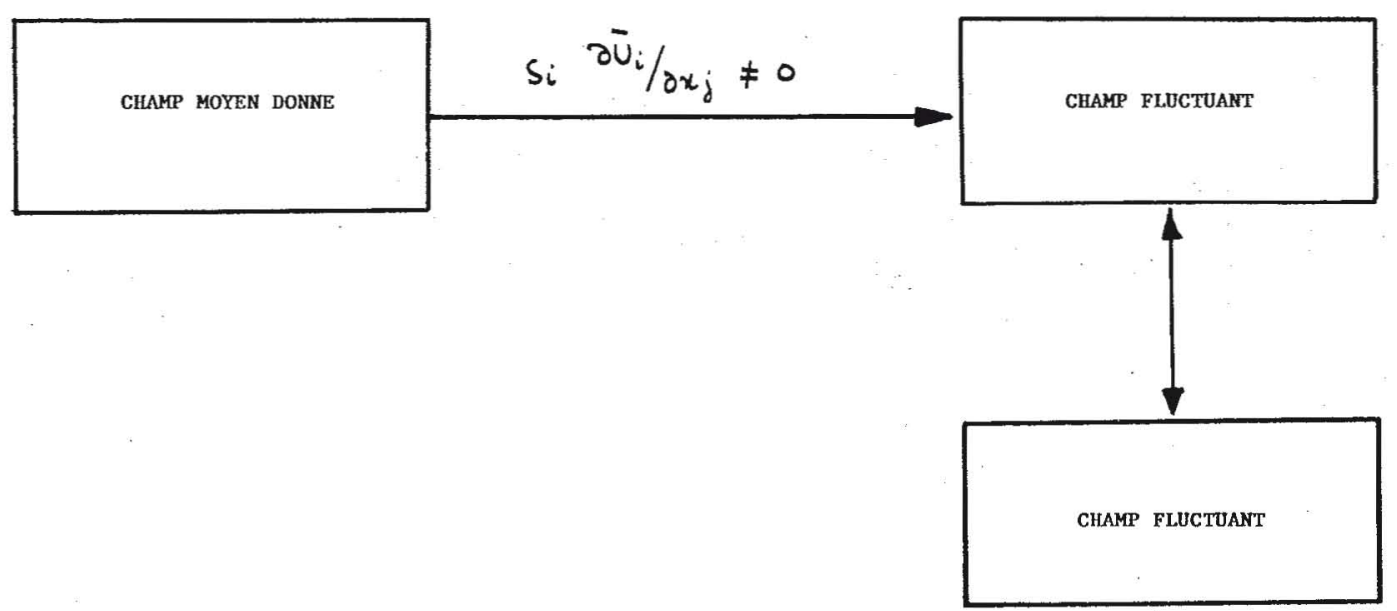

1. Schéma des différentes interactions intervenant dans le modèle de turbulence homogène

C'est dans le but de faciliter l'analyse du phénomène qu'une situation physique simplificatrice fut recherchée, dans laquelle ces divers couplages sont réduits et donc certains mécanismes sont isolés. Le modèle de turbulence homogène permet de satisfaire certains aspects de cette exigence en supposant l'existence d'écoulements turbulents dans lesquels les propriétés statistiques du champ de fluctuations et de leurs diverses dérivées spatiales sont invariantes par translation. En particulier, tous les moments statistiques mettant en jeu un seul point sont indépendants de celui-ci, de sorte que l'on peut écrire par exemple :

$$
\overline{u_{i} u_{j}}=\overline{u_{i} u_{i}}(t) .
$$

Il est, par suite, clair, que le tenseur de Reynolds n'intervient plus dans l'équation (1). D'une manière générale, ce modèle possède les propriétés suivantes :

- Le champ moyen est indépendant du champ fluctuant - L'espace dans lequel se développe l'écoulement est nécessairement infini, compte-tenu de l'invariance par translation.

Toutefois, si l'écoulement moyen évolue sans subir l'action du champ fluctuant, il agit à priori sur celui-ci, par l'intermédiaire de son gradient, comme le montre l'équation du tenseur de Reynolds qui, dans ce cas particulier, se réduit à :

$$
\begin{aligned}
\frac{\partial}{\partial t} \overline{u_{i} u_{i}} & +\overline{u_{i} u_{k}} \cdot \frac{\partial \bar{U}_{j}}{\partial x_{k}}+\overline{u_{i} u_{k}} \cdot \frac{\partial \bar{U}_{i}}{\partial x_{k}} \\
& -\frac{\bar{P} \cdot\left(\frac{\partial u_{i}}{\partial x_{i}}+\frac{\partial u_{j}}{\partial x_{i}}\right)}{\rho}+2 v \cdot \frac{\partial u_{i}}{\partial x_{k}} \cdot \frac{\partial u_{j}}{\partial x_{k}}=0 .
\end{aligned}
$$

Il est clair que les différents termes de cette équation ne doivent pas dépendre de $\vec{x}$ de sorte que le gradient du champ de vitesse moyenne doit être constant spatialement. Nous supposerons en outre que le champ de vitesse moyenne est stationnaire, de sorte que l'équation (1) se réduit à :

$$
\bar{U}_{i} \frac{\partial \bar{U}_{i}}{\partial x_{j}}=-\frac{1}{\rho} \frac{\partial \bar{P}}{\partial x_{i}}
$$

où les composantes $\partial \bar{U}_{i} / \partial x_{i}$ sont constantes et satisfont $\partial \bar{U}_{i} / \partial x_{i}=0$. On peut alors aisément montrer que les seules solutions de ces équations soumises aux contraintes rappelées ci-dessus sont les suivantes :

a) Un champ uniforme (que l'on peut prendre nul par un choix convenable du référentiel galiléen).

b) Un champ induisant un gradient uniforme symétrique (taux de déformation uniforme).

c) Un champ induisant un gradient uniforme antisymétrique (taux de rotation uniforme).

d) Un champ induisant un gradient uniforme plan, dont le cisaillement uniforme est un exemple.

Ainsi, dans ce modèle, le champ stationnaire de vitesse moyenne est donné sous l'une des autre formes rappelées ci-dessus et les interactions entre champs moyens et fluctuants peuvent se schématiser comme sur la figure $I$ :

Par ailleurs, le fait de disposer d'un espace infini pour l'écoulement permet l'analyse de Fourier spatiale du champ de fluctuations de vitesse. On peut ainsi introduire le concept de densité spectrale tridimensionnelle d'énergie $\xi(\vec{k}, t)$, telle que $\xi(\vec{k}, t) \overrightarrow{d k}$ représente l'énergie cinétique moyenne associée au mouvement fluctuant contenue dans l'élément de volume $\overrightarrow{d k}$ centré au point de l'espace des vecteurs d'onde repéré par le vecteur $\vec{k}$. On aura ainsi

$$
\vec{q}^{2} / 2=\frac{1}{2} u_{i} u_{i}=\frac{1}{2}\left(\vec{u}_{i}^{2}+\left(\vec{u}_{2}^{2}+\left(u_{3}^{3}\right)=\int_{k^{3}} \xi(\vec{k}, t) d \vec{k}\right.\right.
$$




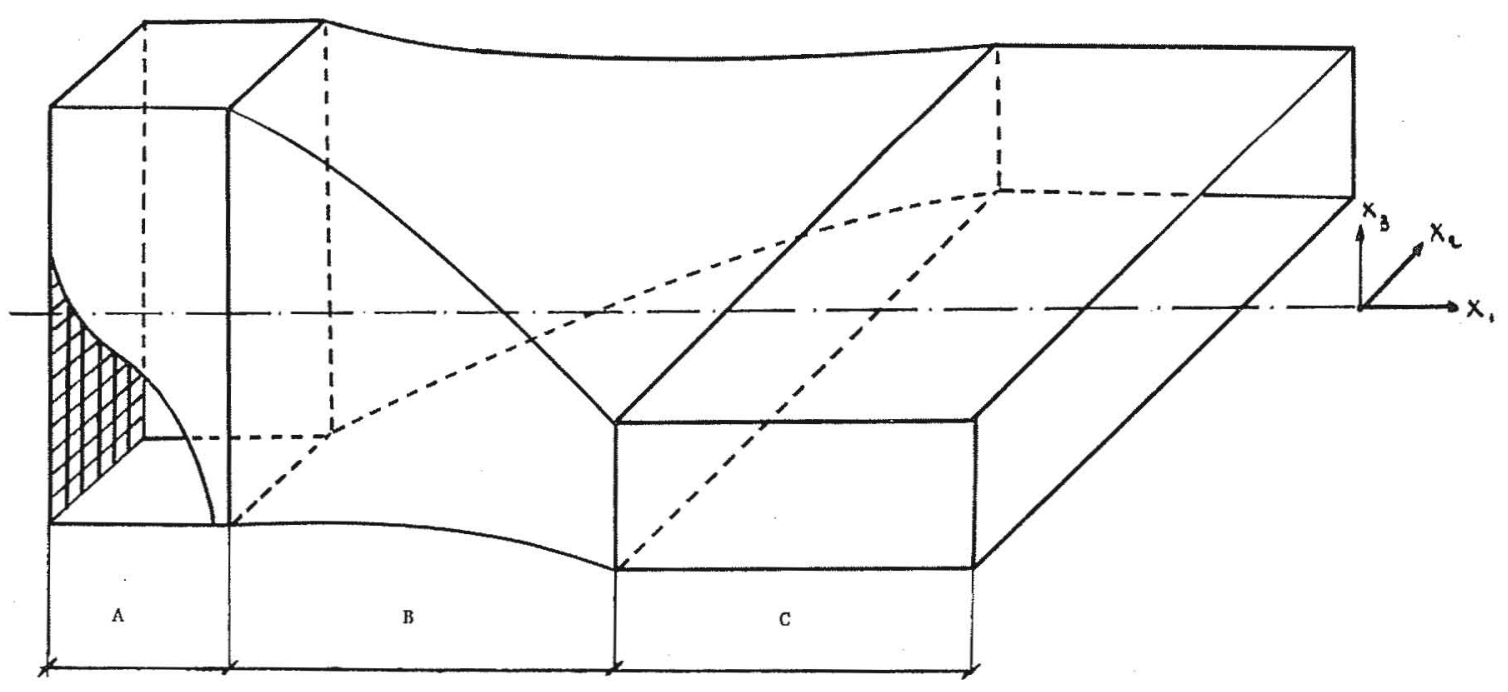

2. Exemple de dispositif expérimental permentam la réalisation d'une turbulence homogène
(A) - Turbulence isotrope générée par une grille

(B) - Distorsion plane (contraction selon $X_{3}$, étirement selon $X_{2}$ )

(C) - Turbulence homogène anisotrope livrée à elle-même
Bien évidemment, ce modèle n'a de véritable intérêt que parce que l'expérience sait le réaliser avec beaucoup d'acuité. Il reste la situation physique de base pour dégager une phénoménologie des principaux mécanismes dominant les écoulements turbulents et, par suite, sert de référence pour tester les méthodes de fermeture des équations de moments statistiques du champ fluctuant. Il sert enfin de cadre pour la mise au point des méthodes de simulation numérique directe des équations de NavierStockes. Il est trivial d'affirmer qu'il est inadapté à la compréhension des mécanismes liés à l'inhomogénéité statistique des écoulements et qui sont fondamentaux dans la naissance de la turbulence, c'est-à-dire la transition. Par conséquent, cette situation physique idéale correspond à une turbulence qui est usuellement qualifiée de "pleinement développée ".

\section{Quelques grands principes de la réalisation expérimentale}

Dans la majorité des expériences approchant cette situation idéale, le mouvement fluctuant est créé à l'aide d'une grille à mailles carrée plane, placée perpendiculairement à la direction de la vitesse moyenne de l'écoulement. Les sillages des différents barreaux de la grille interagissent pour créer à une certaine distance de celle-ci une turbulence qui est statistiquement homogène dans les plans parallèles à la grille dans la zone externe aux couches limites se développant le long des parois (figure $2 \mathrm{~A}$ ). Les propriétés statistiques du mouvement fluctuant ainsi créé évoluent bien évidemment le long de l'axe $\left(0, \vec{x}_{1}\right)$ de l'installation, de sorte que la condition d'homogénéité ne peut être rigoureusement réalisée le long de celui-ci. Ainsi $x_{1}$ devient la variable d'évolution remplaçant le temps $t$ de la théorie à travers la relation de convection $\Delta x_{1}=\bar{U}_{1} \Delta t$. L'homogénéité selon $x_{1}$ est approchée par le fait que l'échelle caractéristique d'évolution des grandeurs selon cette direction est plus grande que l'échelle de corrélation. $\mathrm{Ce}$ principe de génération du champ fluctuant étant acquis, on peut faire subir à ce dernier l'action de différents gradients de vitesse moyenne uniformes en superposant à ce système différents dispositifs adaptés (figure 2B).

\section{Apports du modèle à la phénoménologie de base de la turbulence}

La situation la plus simple que l'on puisse imaginer concerne le cas où le champ moyen est uniforme et, par suite, n'agit pas sur le champ fluctuant qui se trouve livré à lui-même. Les premiers travaux menés dans ce contexte ont conduit au modèle de turbulence homogène et isotrope où le tenseur de Reynolds est isotrope et où $\xi(\vec{k}$, t) ne dépend pas de la direction de $\vec{k}$. On peut alors écrire :

$$
\begin{aligned}
& \overline{u_{i} u_{i}}=\bar{q}^{2} / 3 \delta_{i j} ; \\
& \xi(\vec{k}, t)=\frac{\mathrm{E}(k, t)}{4 \pi k^{2}} \Rightarrow \vec{q}^{2} / 2 \Rightarrow \int_{0}^{\infty} E(k, t) d k \\
& (k=\|\vec{k}\|)
\end{aligned}
$$

Cette situation est la seule qui permet un accès expérimental au spectre $\xi(\vec{k}, t)$ et au terme de transfert qui lui 
est associé qui révèle clairement un échange d'énergie des grosses vers les petites échelles où celle-ci est dissipée ce qui conduit à un déclin de $q^{2}$. Ce point, qui est à la base de la phénoménologie de Kolmogoroff en turbulence tridimensionnelle, conduit en particulier au célèbre comportement en $k^{-5 s}$ du domaine d'inertie des spectres.

En ce qui concerne l'action d'un gradient de vitesse moyenne sur le mouvement fluctuant, la situation la plus simple consiste à faire agir sur une turbulence initiale isotrope une déformation uniforme plane comme cela est schématisée sur la figure $2 \mathrm{~B}$. Conformément à l'intuition, le champ fluctuant, tout en conservant l'homogénéité statistique, devient en plus anisotrope sous l'effet d'un agent extérieur possédant des directions privilégiées qui sont celles d'élongation et de contraction. En outre, on observe une production d'énergie cinétique sous l'influence du champ moyen à gradient symétrique. L'apparition de l'anisotropie s'explique simplement par le fait que les structures tourbillonnaires alignées avec les axes d'élongation et de contraction sont respectivement allongées et raccourcies et par suite induisent dans le plan perpendiculaire à leur axe, des vitesses qui sont augmentées dans le premier cas et diminuées dans l'autre. La production d'énergie s'explique aussi par cette phénoménologie car l'allongement des tourbillons est toujours plus "efficace" que leur contraction.

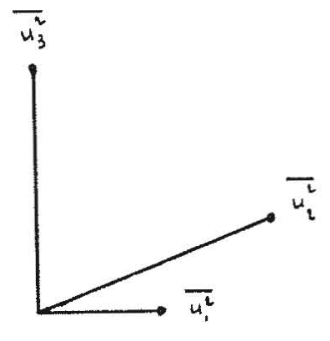

$(\Lambda)$

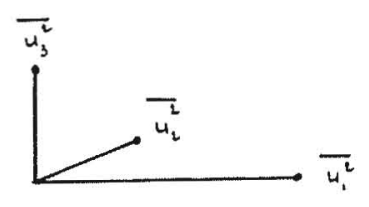

(B)
3. Exemples de répartition d'énergie entre les composantes principales du tenseur de Reinolds dans une turbulence homogène anisotrope.

Adresses de l'auteur

Monsieur J.N. Gence

Laboratoire de mécanique des fluides - Ecole Centrale de Lion 36. Arenue Guy de Colloque - 69130 - Ecully Cedex oll

I.S.I.D.T. - Université de Lyon I - 69622 - Villeurbanne Cedex.

\section{Analyses récentes}

En ce qui concerne la seule interaction du champ fluctuant (donc en absence de gradient de vitesse moyenne), on peut imaginer une situation plus générale que le cas de la turbulence isotrope en considérant une turbulence homogène mais anisotrope livrée à elle-même. Une telle situation s'observe par exemple dans la partie $\mathrm{C}$ du conduit de la figure 2 où la turbulence ayant été rendue anisotrope sous l'effet de la distorsion, évolue dans un champ uniforme de vitesse moyenne. On retrouve, bien évidemment, dans cette situation le phénomène de déclin visqueux observé dans le cas de la turbulence isotrope puisque l'on est en face d'un phénomène dissipatif non alimenté en énergie mais, en outre, l'interaction du champ fluctuant se traduit par une tendance au retour vers l'isotropie qui conduit à une équipartition de l'énergie entre les composantes principales du tenseur de Reynolds. La modélisation de ce phénomène, fondamentale pour les méthodes de prédiction dans l'espace physique, nécessite la réponse à la question suivante : la turbulence anisotrope livrée à elle-même retourne-t-elle plus vite vers l'isotropie qu'elle ne perd de l'énergie ou plus lentement? Des travaux récents montrent qu'en fait ceci dépend de la manière dont est répartie l'anisotropie entre les composantes principales du tenseur de Reynolds. En particulier, dans la situation (A) de la figure 3, le retour vers l'isotropie est trois fois plus rapide que le déclin d'énergie alors que dans le cas (B) les deux mécanismes ont des temps caractéristiques identiques. Des études de l'anisotropie des différentes échelles ainsi que des simulations numériques doivent permettre de dégager une phénoménologie claire de ce mécanisme.

Les autres études récentes menées dans le contexte de la turbulence homogène, à la fois sur le plan expérimental et sur celui des simulations numériques, concernent l'action d'une rotation de solide sur une turbulence initiale isotrope. Le gradient moyen est ici antisymétrique, de sorte qu'il n'alimente pas le mouvement fluctuant en énergie. Les résultats de ces études encore en cours révèlent que l'énergie fluctuante décline d'autant plus lentement que le taux de rotation est élevé. Par ailleurs, le tenseur de Reynolds reste sensiblement isotrope mais une forte anisotropie des échelles s'installe de sorte que la turbulence cesse d'être isotrope.

\section{Conclusions}

Le modèle de turbulence homogène est le seul qui permet l'isolation de l'interaction du champ fluctuant. Par ailleurs, lorsqu'un champ de vitesse moyenne agit, il permet le choix du gradient uniforme qui lui est associé. En outre, comme il ne fait pas intervenir de limites, il est particulièrement adapté à la mise au point des méthodes de fermeture et à la simulation numérique en général. Toutes ces propriétés font que c'est dans son contexte que la théorie de la turbulence est la plus développée. 